\title{
BMJ Open Analgesia dose prescribing and estimated glomerular filtration rate decline: a general practice database linkage cohort study
}

\author{
Paul Nderitu, ${ }^{1}$ Lucy Doos, ${ }^{2}$ Vicky Y Strauss, ${ }^{3}$ Mark Lambie, ${ }^{1}$ Simon J Davies, ${ }^{1}$ \\ Umesh T Kadam ${ }^{1}$
}

To cite: Nderitu P, Doos L, Strauss VY, et al. Analgesia dose prescribing and estimated glomerular filtration rate decline: a general practice database linkage cohort study. BMJ Open 2014;4:e005581.

doi:10.1136/bmjopen-2014005581

- Prepublication history for this paper is available online. To view these files please visit the journal online (http://dx.doi.org/10.1136/ bmjopen-2014-005581).

Received 28 April 2014 Revised 29 July 2014 Accepted 30 July 2014

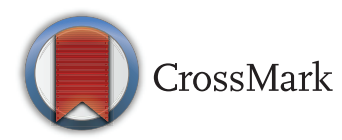

${ }^{1}$ Health Services Research Unit, Institute of Science and Technology in Medicine, Keele University, Keele, UK ${ }^{2}$ Public Health, Epidemiology and Biostatistics, NIHR Horizon Scanning Centre, School of Health and Population Sciences, University of Birmingham, Birmingham, UK

${ }^{3}$ Nuffield Department of Orthopaedics, Rheumatology and Musculoskeletal

Sciences, Centre for Statistics in Medicine, Botnar Research Centre, University of Oxford, Oxford, UK

Correspondence to Dr Paul Nderitu; p.nderitu@doctors.org.uk

\section{ABSTRACT}

Objective: We aimed to quantify the short-term effect of non-steroidal anti-inflammatory drugs (NSAIDs), aspirin and paracetamol analgesia dose prescribing on estimated glomerular filtration rate (eGFR) decline in the general practice population.

Design: A population-based longitudinal clinical data linkage cohort study.

Setting: Two large general practices in North Staffordshire, UK.

Participants: Patients aged 40 years and over with $\geq 2$ eGFR measurements spaced $\geq 90$ days apart between 1 January 2009 and 31 December 2010 were selected.

Exposure: Using WHO Defined Daily Dose standardised cumulative analgesia prescribing, patients were categorised into non-user, normal and high-dose groups. Outcome measure: The primary outcome was defined as a $>5 \mathrm{~mL} / \mathrm{min} / 1.73 \mathrm{~m}^{2} /$ year eGFR decrease between the first and last eGFR. Logistic regression analyses were used to estimate risk, adjusting for sociodemographics, comorbidity, baseline chronic kidney disease (CKD) status, renin-angiotensin-system inhibitors and other analgesia prescribing.

Results: There were 4145 patients (mean age 66 years, $55 \%$ female) with an analgesia prescribing prevalence of $17.2 \%$ for NSAIDs, $39 \%$ for aspirin and $22 \%$ for paracetamol and stage 3-5 CKD prevalence was $16.1 \%$ $(\mathrm{n}=667)$. Normal or high-dose NSAID and paracetamol prescribing was not significantly associated with eGFR decline. High-dose aspirin prescribing was associated with a reduced risk of eGFR decline in patients with a baseline (first) eGFR $\geq 60 \mathrm{~mL} / \mathrm{min} / 1.73 \mathrm{~m}^{2} ; \mathrm{OR}=0.52$ (95\% Cl 0.35 to 0.77 ).

Conclusions: NSAID, aspirin and paracetamol prescribing over 2 years did not significantly affect eGFR decline with a reduced risk of eGFR decline in high-dose aspirin users with well-preserved renal function. However, the long-term effects of analgesia use on eGFR decline remain to be determined.

\section{BACKGROUND}

Chronic kidney disease (CKD) is a major cause of morbidity and mortality worldwide ${ }^{1}$

\section{Strengths and limitations of this study}

- General practice patients with a wide spectrum of chronic kidney disease (CKD) and non-CKD were included in this study.

- Analgesia dose prescribing was standardised using the WHO defined daily dose method.

- The outcome of estimated glomerular filtration rate decline is based on current clinical guidelines and changes to renal function were correlated against analgesia use.

- Data on over-the-counter use was not available in this study.

- There were a limited number of patients with stage 3-5 CKD with high-dose analgesia use.

requiring risk factors of CKD progression to be identified and minimised. ${ }^{2}$ Comorbidities such as diabetes mellitus (DM), cardiovascular disease (CVD) and hypertension are wellknown risk factors for CKD progression. ${ }^{1}$ The National Institute for Health and Clinical Excellence (NICE) UK guidelines (2008) identified non-steroidal antiinflammatory drugs (NSAIDs) as one possible risk factor for CKD progression and advised that their use be restricted in CKD patients. ${ }^{2}$ Although the acute effects of NSAID use on renal function are well recognised, ${ }^{3}$ the chronic effects are unclear with conflicting findings in the literature. Few studies have used standardised drug doses to quantify the unsafe levels of use with invalid or arbitrary definitions of maintenance or high levels of analgesic use. ${ }^{4}$

NSAIDs and aspirin in comorbid cardiometabolic diseases are used widely in the CKD and general population with prescriptions making up a significant proportion of use. ${ }^{5}$ Low-dose aspirin is indicated for use as a thromboprophylactic agent while NSAIDs are indicated for use as simple analgesics ${ }^{6}$ by patients with a range of musculoskeletal 
pathology. Therefore, the complete avoidance of NSAIDs or aspirin among CKD patients may impact on quality of life by excluding a major group of drug treatment for pain symptoms. NSAIDs have been associated with gastrointestinal and vascular complications; ${ }^{7}$ therefore, where contraindicated, paracetamol is often the preferred simple analgesic. However, paracetamol (acetaminophen) is a metabolite of the banned nephrotoxic NSAID phenacetin and has been associated with renal dysfunction ${ }^{8} 9$ with limited evidence on its effect on estimated glomerular filtration rate (eGFR) decline. ${ }^{8}{ }^{10} \mathrm{Few}$ studies have examined the effect of NSAIDs, aspirin and paracetamol prescribing on eGFR decline among the CKD population. ${ }^{4}$

Given the clinical importance of CKD, the widespread use of NSAIDs, aspirin and paracetamol and the conflicting literature, further research is required into the effects of analgesia use on eGFR decline. This study aimed to investigate the effect of normal and high dose analgesia prescribing on eGFR decline in the general practice population with the aim of aiding prescribing decision-making among patients with CKD requiring the aforementioned analgesics.

\section{METHODS}

\section{Study design and setting}

Patients were selected from two general practices, both of which contribute to the Consultations in Primary Care Archive (CiPCA) and Prescriptions in Primary Care Archive (PiPCA) interlinked databases. The anonymised databases contain routinely collected consultation and prescription data recorded since 2000 from 13 general practices in North Staffordshire, UK. ${ }^{11}{ }^{12}$ Practices undergo annual assessments, feedback and training on the quality of morbidity recording. ${ }^{13}$ In addition to

Figure 1 Flow chart of the patient selection process. consultation (containing diagnostic data) and prescription (containing prescribed analgesia and co-drug therapies) data between 2009 and 2010, laboratory data (containing blood tests) for all included patients in the two included general practices $(\mathrm{n}=23028$ in 2009) chosen were available at the time of the study. ${ }^{11}{ }^{12}$ Ethical approval for CiPCA was granted by the North Staffordshire Ethics Committee.

\section{Cohort selection}

Patients aged 40 years and over with at least one eGFR measurement from 1 January 2009 to 31 December 2010 were identified (2-year study period). Of these, patients with two or more eGFR measurements during the 2-year study period were eligible for inclusion and formed the study cohort (figure 1). Patients aged under 40 years on 1 January 2009 were excluded because stage 3-5 CKD prevalence is low in this age group. ${ }^{14}$

Patients with less than two valid eGFR measurements were excluded as eGFR decline could not be calculated. Patients with less than 90 days between the first and last eGFR measurements were also excluded to minimise the impact of acute renal failure and to gain more accurate estimates of eGFR decline and minimise the effects of measurement variability. Therefore, patients had a maximum follow-up interval of up to 2 years between the first and last eGFR and a minimal follow-up of 90 days. Only the first and last eGFR measurements during the study period were used to calculate the eGFR decline rate.

\section{Measurement of renal function}

The eGFR was calculated using the simplified 4-variable Modification of Diet in Renal Disease (MDRD) equation, which includes the variables for serum creatinine, age, gender and available ethnicity data. ${ }^{1}$ As defined by

\begin{tabular}{|c|c|}
\hline $\begin{array}{l}\text { Practice Population } \\
\qquad \begin{array}{l}\text { (2 practices) } \\
\mathbf{N}=\mathbf{2 3 , 0 2 8}\end{array}\end{array}$ & Excluded \\
\hline 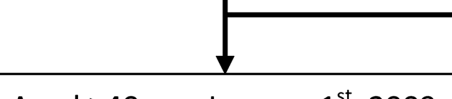 & $\begin{array}{l}\text { inclusion } \\
\mathbf{N}=\mathbf{9 , 9 4 7}\end{array}$ \\
\hline $\begin{array}{l}\text { Aged } \geq 40 y \text { on January } 1,2009 \\
\qquad N=13,081\end{array}$ & Excluded \\
\hline & $\begin{array}{c}0 \text { eGFR } \\
\text { measurements } \\
\mathbf{N}=\mathbf{5 , 4 2 4}\end{array}$ \\
\hline $\begin{array}{l}\geq 1 \text { valid eGFR measurements } \\
\text { during } \\
\text { Jan- } 1^{\text {st }}-2009 \text { to } D e c-31^{\text {st }}-2010 \\
N=7,657\end{array}$ & $\begin{array}{c}\frac{\text { Excluded }}{<2 \text { eGFR }} \\
\text { measurements }\end{array}$ \\
\hline $\mathbf{v}$ & First and last eGFR \\
\hline $\begin{array}{l}\geq 2 \text { valid eGFR measurements } \\
\qquad \mathbf{N}=\mathbf{4 , 1 4 5}\end{array}$ & $\begin{array}{c}<90 \text { days apart } \\
\mathbf{N}=\mathbf{4 6 1}\end{array}$ \\
\hline
\end{tabular}


the National Kidney Foundation-Kidney Disease Outcomes Quality Initiatives (NKF-KDOQI) guidelines, ${ }^{1}$ CKD is categorised into five stages (table 1 ). Stage 3-5 CKD can be categorised from the eGFR alone ${ }^{1}$ but stage 1 and 2 CKD requires further evidence of renal pathology, ${ }^{1}$ which was not available in our study. Therefore, an approach employed by de Lusignan et $a l^{14}$ was used instead of stage 1 and 2 CKD definitions whereby patients with baseline (first) eGFRs between 60 and 89 were categorised as 'mildly impaired' and those with baseline eGFRs $\geq 90$ were categorised as 'normal'. ${ }^{14}$

\section{Cumulative exposure definition}

Prescribed analgesics were identified by means of the relevant British National Formulary chapters. ${ }^{6}$ Data on drug dose, frequency and quantity (tablets or millilitres) for each drug were sought. All prescriptions (both continuously and non-continuously issued) for analgesics given to patients from 1 January 2009 to the last eGFR measurement date (exposure period) were included in the cumulative exposure. Cumulative exposure before the last eGFR measurement was calculated as follows:

Cumulative exposure $=$ Total tablets or millilitres

(before the last eGFR measurement)

$\times$ drug strength per tablet or millilitre

Cumulative drug exposure was standardised using the WHO anatomical therapeutic classification and defined daily dose (DDD) method. Cumulative DDDs prescribed per drug for each subject were then calculated as follows:

Cumulative DDD = Cumulative exposure/DDD per drug.

Patients were categorised into non-user (0 DDDs), normal-dose (DDDs <85th centile) and high-dose (DDDs $\geq 85$ th centile) users groups per drug based on prescribed analgesics. The chosen cut-offs for normal and high-dose analgesia prescribing are based on the Gooch et $a l,{ }^{16}$ study which employed methodologies

\begin{tabular}{|c|c|c|}
\hline $\begin{array}{l}\text { CKD } \\
\text { Stage }\end{array}$ & Description & $\begin{array}{l}\text { GFR }(\mathrm{mL} / \mathrm{min} / \\
\left.1.73 \mathrm{~m}^{2}\right)\end{array}$ \\
\hline 1 & $\begin{array}{l}\text { Kidney damage with normal } \\
\text { or increased GFR }\end{array}$ & $\geq 90$ \\
\hline 2 & $\begin{array}{l}\text { Kidney damage with mild } \\
\text { reduction in GFR }\end{array}$ & 60-89 \\
\hline 3 & Moderate reduction in GFR & $30-59$ \\
\hline 4 & Severe reduction in GFR & $15-29$ \\
\hline 5 & ESRD & $<15$ (or dialysis) \\
\hline
\end{tabular}

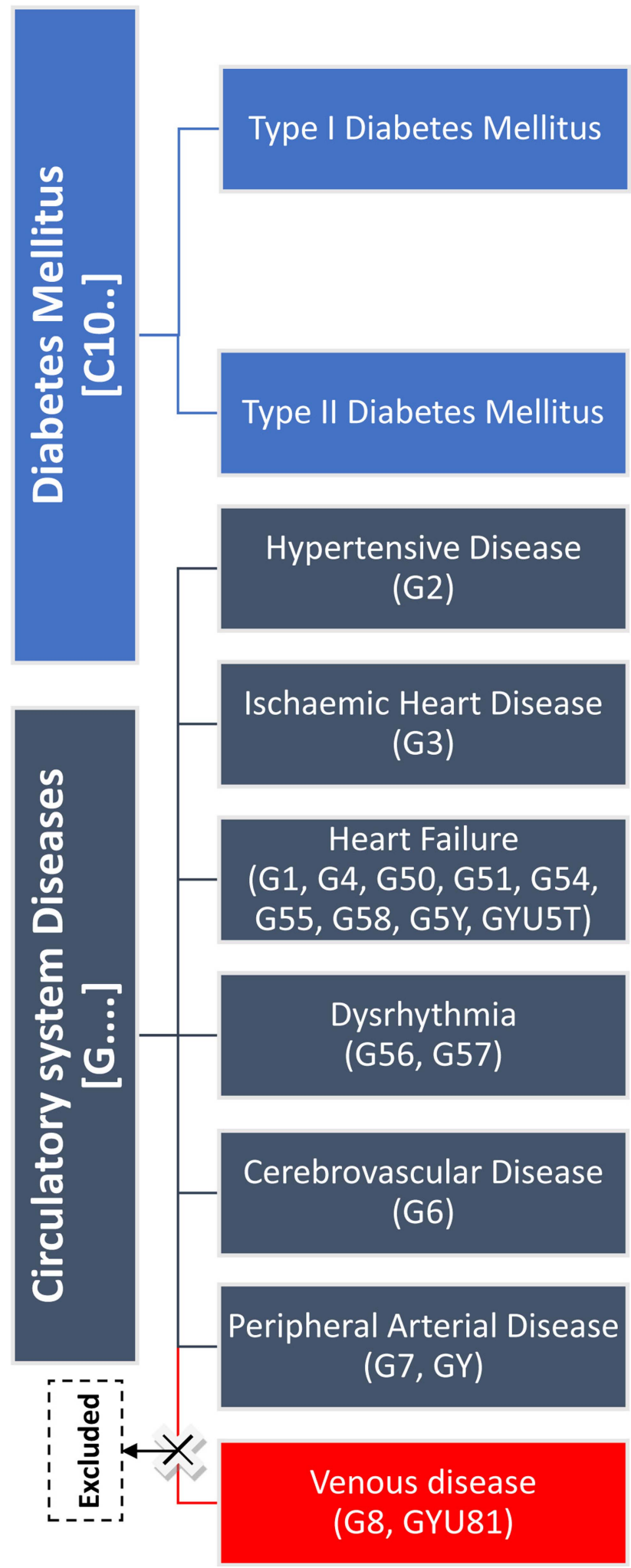

Figure 2 Included and excluded diabetes mellitus and chronic kidney disease pathologies using READ classification codes.

similar to our study and are adjusted for differences in study duration.

In this study, all prescribed analgesics were assumed to have been taken by the patient. Prescriptions given 
between 1 January 2009 and the date of the first eGFR were included as high dose analgesia use during this time may have had subsequent effects on renal function decline which would affect the subsequent eGFR decline rate.

\section{Outcome definition}

The outcome of eGFR decline was defined in this study as a change of $>5 \mathrm{~mL} / \mathrm{min} / 1.73 \mathrm{~m}^{2}$ per year in accordance with the UK NICE (2008) and Kidney DiseaseImproving Global Outcomes (KDIGO 2012) guidelines. ${ }^{2}{ }^{17}$ Non-significant eGFR decline was defined as a change of $\leq 5 \mathrm{~mL} / \mathrm{min} / 1.73 \mathrm{~m}^{2}$ per year. The yearly eGFR decline rate was calculated as follows:

eGFR decline rate per year $=($ last eGFR - first eGFR $)$

$\times(365 /$ eGFR interval in days $)$.

\section{Covariates}

\section{Socio-demographics}

Socio-demographic data included age, gender and deprivation quartiles. Patients were given a deprivation score based on the England indices of multiple deprivation (IMD) score (2007) and placed into quartiles relative to their individual scores. The IMD is used widely to assess deprivation in the UK. ${ }^{18}$

\section{Comorbidity}

Comorbidity, defined as the presence of DM (type I and II) or Cardiovascular Disease (CVD) at any point up to 31 December 2010, was identified from the CiPCA database using the relevant unique clinical codes (READ codes). Subjects with READ codes [C10..] and all daughter codes were defined as having DM. CVD status included the $[G \ldots .$.$] READ codes and all daughter codes$ including those for hypertensive disease, ischaemic heart disease, heart failure, dysrhythmia, cerebrovascular disease and peripheral arterial disease; codes for venous disease were excluded (see figure 2).

\section{Codrug therapy}

Renin-angiotensin system inhibitors (RAS-i) prescribing was defined as the prescription of one or more ACE inhibitors or angiotensin receptor blockers or renin inhibitors during the 2-year study period.

The chosen covariates are clinically important, ${ }^{2}$ can augment renal function decline ${ }^{1}$ and feature prominently in recent studies. ${ }^{8} 10$ 19-21

\section{Statistical methods}

Descriptive analyses are presented as means and SD for normally distributed continuous variables, medians and IQR for skewed continuous data and percentages for dichotomous variables. Parametric tests for significant differences were determined using analysis of variance, Welch's $\mathrm{F}$ or $\chi^{2}$ tests where appropriate.

Logistic regression analyses, using 95\% CIs, were used to assess the association between analgesia dose
Table 2 Characteristics for patients with single versus multiple eGFR measurements

\begin{tabular}{|c|c|c|}
\hline $\begin{array}{l}\text { Baseline } \\
\text { characteristics }\end{array}$ & $\begin{array}{l}\text { Single eGFR } \\
\text { measurement } \\
n=7657\end{array}$ & $\begin{array}{l}\text { Multiple eGFR } \\
\text { measurements } \\
n=4145\end{array}$ \\
\hline Age, mean (SD) & $64(13)$ & $66(12)$ \\
\hline \multicolumn{3}{|l|}{ Gender, \% } \\
\hline Male & 44.8 & 45.4 \\
\hline Female & 55.2 & 54.6 \\
\hline \multicolumn{3}{|l|}{ Deprivation, \%* } \\
\hline Least deprived & 25.8 & 24.0 \\
\hline Q2 & 24.7 & 25.2 \\
\hline Q3 & 25.9 & 26.7 \\
\hline Most deprived & 23.6 & 24.1 \\
\hline NSAID use, \%* & 17.3 & 17.2 \\
\hline Aspirin use, \%* & 27.8 & 38.8 \\
\hline $\begin{array}{l}\text { Paracetamol use, } \\
\%^{*}\end{array}$ & 17.1 & 21.9 \\
\hline CVD, \% & 52.7 & 69.0 \\
\hline $\mathrm{DM}, \%$ & 17.3 & 26.4 \\
\hline RAS-i, \% & 40.2 & 53.9 \\
\hline $\begin{array}{l}\text { Baseline eGFR, } \\
\text { Median[ IQR]† }\end{array}$ & 84 [70-91] & 83 [68-91] \\
\hline \multicolumn{3}{|c|}{$\begin{array}{l}\text { *Prescribed before the last eGFR measurement. } \\
\text { †First eGFR. } \\
\text { CVD, cardiovascular disease; DM, diabetes mellitus; NSAID, } \\
\text { non-steroidal anti-inflammatory drug; RAS-I, renin-angiotensin } \\
\text { system inhibitors. }\end{array}$} \\
\hline
\end{tabular}

prescribing and eGFR decline with non-users of the respective drugs acting as a reference group. Models were adjusted for sociodemographic status, comorbidity, codrug therapy, other analgesia prescribing (eg, aspirin or paracetamol use among NSAID users) and baseline CKD stage. Patients were divided into normal or mildly impaired eGFR and stage 3-5 CKD subgroups using the first eGFR (baseline eGFR). Logistic regression was used as the outcome was binary and this study explored the magnitude of association. Statistical analyses were carried out on SPSS, V.20.0, IBM, USA.

\section{RESULTS}

\section{Study population characteristics}

There were 23028 registered patients in the chosen practices of which 7657 had at least one eGFR measurement during the study period. Of these, 4145 eligible patients with two or more eGFR measurements were included with a mean age of 66 years (SD 12 years) and $54.6 \%$ were women. Characteristics of patients with single versus multiple eGFR measurements are shown in table 2 demonstrating the greater burden of comorbidity as well as aspirin and paracetamol use in patients with multiple eGFR measurements.

Analgesia prescribing prevalence was $17.2 \%$ (normal dose $=605$, high dose $=106$ ) for NSAIDs, $38.8 \%$ (normal dose $=1837$, high dose $=222$ ) for aspirin and $21.9 \%$ (normal dose $=758$, high dose $=150$ ) for paracetamol. The average prescribed NSAID doses were 0.18 DDD 
Table 3 WHO DDD index (2012) for included NSAIDs, aspirin and paracetamol

\begin{tabular}{ll}
\hline Analgesic & WHO DDD in grams \\
\hline Aceclofenac & 0.2 \\
Acemetacin & 0.12 \\
Aspirin & 0.075 \\
Dexibuprofen & 0.8 \\
Diclofenac & 0.1 \\
Etodolac & 0.4 \\
Etoricoxib & 0.060 \\
Flurbiprofen & 0.2 \\
Ibuprofen & 1.2 \\
Indometacin & 0.1 \\
Mefenamic acid & 1 \\
Meloxicam & 0.015 \\
Nabumetone & 1 \\
Naproxen & 0.5 \\
Paracetamol & 3 \\
Phelylbutazone & 0.3 \\
Piroxicam & 0.020 \\
Tiaprofenic acid & 0.6 \\
\hline NSAIDs, non-steroidal anti-inflammatory & drug; DDD, defined daily \\
dose. &
\end{tabular}

per day for normal and 1.21 DDD per day for high dose NSAID users, equivalent to $0.22 \mathrm{~g}$ and $1.45 \mathrm{~g}$ per day, respectively, of ibuprofen. The average prescribed aspirin doses were 0.81 DDD (61 mg) per day for normal and 1.44 DDD (110 mg) per day for high-dose aspirin users. Average prescribed paracetamol doses were 0.14 DDD $(0.42 \mathrm{~g})$ per day for normal and 0.65 DDD (1.95 g) per day for high-dose paracetamol users. Table 3 shows the DDD for all included NSAIDs, aspirin and paracetamol.

The prevalence of DM was $26.4 \% \quad(n=1094)$ and $69.0 \%(\mathrm{n}=2859)$ for CVD, however, the majority of patients $(55 \%)$ had hypertension. RAS-i prescribing prevalence was $53.9 \%(\mathrm{n}=2236)$. The median [IQR] number of eGFR measurements was 3 [2-4] and the mean time between first and last eGFR measurements (SD) was 270 (125) days. The median baseline (first) eGFR was 83 [68-91], baseline stage 3-5 CKD prevalence was $16.1 \%$ ( $\mathrm{n}=667$, females $19.1 \%$; males $12.5 \%)$ and the incidence of eGFR decline was 26.5\% ( $n=1099)$. Characteristics of patients with significant and nonsignificant eGFR decline are shown in table 4 .

\section{Characteristics of analgesia users and non-users}

Compared to non-NSAID users, NSAID users were significantly younger, less likely to be prescribed aspirin but more likely to be prescribed paracetamol (table 5). NSAID users also had significantly lower levels of CVD and RAS-i prescribing than non-NSAID users. Gender,

Table 4 Characteristics of patients with significant and non-significant eGFR decline

\begin{tabular}{|c|c|c|c|}
\hline Baseline characteristics & $\begin{array}{l}\text { Non-significant } \\
\text { eGFR decline } \\
\mathrm{n}=3046\end{array}$ & $\begin{array}{l}\text { Significant } \\
\text { eGFR decline } \\
n=1099\end{array}$ & p Value \\
\hline Age, mean (SD) & $66(12)$ & $68(12)$ & $<0.001^{*}$ \\
\hline \multicolumn{4}{|l|}{ Gender, \% } \\
\hline Male & 44.9 & 47.0 & \multirow[t]{2}{*}{0.237} \\
\hline Female & 55.1 & 53.0 & \\
\hline \multicolumn{4}{|l|}{ Deprivation, \%† } \\
\hline Least deprived & 24.2 & 23.5 & \multirow[t]{4}{*}{0.939} \\
\hline Q2 & 25.0 & 25.8 & \\
\hline Q3 & 26.6 & 26.9 & \\
\hline Most deprived & 24.2 & 23.8 & \\
\hline NSAID use, \% $\%$ & 17.7 & 15.6 & 0.367 \\
\hline Aspirin use, $\% \ddagger$ & 38.5 & 39.6 & 0.545 \\
\hline Paracetamol use, \%‡ & 22.0 & 21.6 & 0.869 \\
\hline CVD, \% $(n=2859) \S$ & 68.2 & 71.2 & 0.058 \\
\hline Hypertension, \% $(n=2259)$ & 54.3 & 55.1 & 0.669 \\
\hline Ischaemic heart disease, $\%(n=642)$ & 14.7 & 17.7 & $0.021^{*}$ \\
\hline Heart failure, \% (128) & 2.4 & 5.0 & $<0.001^{*}$ \\
\hline Peripheral vascular disease, $\%(n=63)$ & 1.3 & 2.1 & 0.070 \\
\hline Cerebrovascular disease, $\%(n=297)$ & 7.0 & 7.7 & 0.394 \\
\hline Dysrhythmia, \% $(n=141)$ & 3.0 & 4.5 & $0.014^{*}$ \\
\hline $\mathrm{DM}, \%(n=1094)$ & 26.5 & 26.2 & 0.237 \\
\hline RAS-i, \% $(n=2236)$ & 53.3 & 55.9 & 0.135 \\
\hline
\end{tabular}


Table 5 Characteristics of NSAID, aspirin and paracetamol users and non-users

\begin{tabular}{|c|c|c|c|c|c|c|c|c|c|c|c|c|}
\hline \multirow[b]{2}{*}{ Baseline characteristics } & \multicolumn{3}{|c|}{ NSAID use } & \multirow[b]{2}{*}{$p$ Value } & \multicolumn{3}{|c|}{ Aspirin use } & \multirow[b]{2}{*}{ p Value } & \multicolumn{3}{|c|}{ Paracetamol use } & \multirow[b]{2}{*}{ p Value } \\
\hline & $\begin{array}{l}\text { None } \\
(n=3434)\end{array}$ & $\begin{array}{l}\text { Normal } \\
(n=605)\end{array}$ & High $(n=106)$ & & $\begin{array}{l}\text { None } \\
(n=2536)\end{array}$ & $\begin{array}{l}\text { Normal } \\
(n=1387)\end{array}$ & High $(n=222)$ & & $\begin{array}{l}\text { None } \\
(n=3237)\end{array}$ & $\begin{array}{l}\text { Normal } \\
(n=758)\end{array}$ & High $(n=150)$ & \\
\hline Age, mean (SD) & $67(12)$ & $62(12)$ & $61(10)$ & $<0.001^{*}$ & $64(12)$ & $70(11)$ & $71(10)$ & $<0.001^{\star}$ & $65(12)$ & $71(11)$ & $72(12)$ & $<0.001^{*}$ \\
\hline \multicolumn{13}{|l|}{ Gender, \% } \\
\hline Male & 46.0 & 43.3 & 39.6 & 0.227 & 40.5 & 53.5 & 51.8 & $<0.001^{\star}$ & 46.5 & 42.5 & 38.0 & $0.025^{*}$ \\
\hline Female & 54.0 & 56.7 & 60.4 & & 59.5 & 46.5 & 48.2 & & 53.5 & 57.5 & 62.0 & \\
\hline \multicolumn{13}{|l|}{ Deprivation, \%† } \\
\hline Least deprived & 24.2 & 24.0 & 17.9 & 0.289 & 25.1 & 22.0 & 23.1 & $0.013^{*}$ & 25.5 & 19.9 & 12.7 & $<0.001^{*}$ \\
\hline Q2 & 24.8 & 27.1 & 27.4 & & 25.8 & 24.1 & 25.8 & & 25.3 & 24.3 & 28.0 & \\
\hline Q3 & 26.6 & 27.8 & 24.5 & & 26.9 & 26.9 & 23.1 & & 26.2 & 28.8 & 26.7 & \\
\hline Most deprived & 24.4 & 21.2 & 30.2 & & 22.2 & 27.0 & 28.1 & & 23.0 & 27.0 & 32.7 & \\
\hline Cumulative DDDs, Median [IQR]‡ & 0 & $49[28-126]$ & $717[560-908]$ & $\mathrm{N} / \mathrm{A}$ & 0 & $448[252-560]$ & 840 [728-1120] & N/A & 0 & $50[17-133]$ & $383[315-517]$ & $\mathrm{N} / \mathrm{A}$ \\
\hline NSAID use, $\% \S$ & N/A & $\mathrm{N} / \mathrm{A}$ & $N / A$ & & 19.5 & 13.2 & 14.9 & $<0.001^{*}$ & 16.4 & 20.2 & 18.0 & $0.044^{*}$ \\
\hline Aspirin use, $\% \S$ & 40.6 & 31.1 & 26.4 & $<0.001^{*}$ & N/A & N/A & $\mathrm{N} / \mathrm{A}$ & & 37.9 & 45.0 & 52.0 & $<0.001^{*}$ \\
\hline Paracetamol use, \%§ & 21.2 & 24.8 & 28.3 & $0.039^{\star}$ & 19.4 & 25.0 & 30.6 & $<0.001^{*}$ & $\mathrm{~N} / \mathrm{A}$ & $\mathrm{N} / \mathrm{A}$ & $\mathrm{N} / \mathrm{A}$ & \\
\hline CVD, \% & 71.3 & 58.3 & 54.7 & $<0.001^{*}$ & 60.7 & 81.8 & 82.9 & $<0.001^{*}$ & 68.6 & 69.5 & 74.0 & 0.354 \\
\hline $\mathrm{DM}, \%$ & 26.9 & 23.6 & 25.5 & 0.237 & 20.3 & 36.5 & 32.4 & $<0.001^{*}$ & 25.5 & 29.4 & 29.3 & 0.066 \\
\hline RAS-i, \% & 55.9 & 45.6 & 37.7 & $<0.001^{*}$ & 47.0 & 64.7 & 65.8 & $<0.001^{*}$ & 53.9 & 52.0 & 64.0 & $0.026^{*}$ \\
\hline $\begin{array}{l}\text { Yearly eGFR decline, Median } \\
\text { [IQR] }\end{array}$ & $\begin{array}{l}0[-5.59 \\
+0.84]\end{array}$ & $0[-4.93,0]$ & $0[-4.18,+1.24]$ & 0.571 & $\begin{array}{l}0[-5.43 \\
+0.27]\end{array}$ & $\begin{array}{l}0[-5.62 \\
+0.84]\end{array}$ & $0[-4.01,+2.56]$ & $0.028^{\star}$ & $\begin{array}{l}0[-5.43 \\
+0.65]\end{array}$ & $\begin{array}{l}0[-5.29 \\
+1.69]\end{array}$ & $-0.96[-5.49,0]$ & 0.148 \\
\hline
\end{tabular}


deprivation, DM and eGFR decline rates did not significantly differ between NSAID users and non-NSAID users.

Aspirin users were significantly older, more likely to be male with higher levels of deprivation than non-aspirin users. They were also less likely to use NSAIDs but more likely to use paracetamol than non-aspirin users. CVD, DM and RAS-i prescribing were significantly higher among aspirin users than non-aspirin users. The eGFR decline rate distributions differed significantly between normal, high dose and non-users of aspirin $(p=0.028)$.

Paracetamol users were significantly older, more likely to be female with higher levels of deprivation compared to non-paracetamol users. They were also significantly more likely to be prescribed NSAIDs, aspirin or RAS-i than non-paracetamol users. The comorbidity status and eGFR decline rates did not differ significantly between paracetamol users and non-paracetamol users.

\section{Associations between analgesia, dose and chronic kidney disease progression}

Normal or high dose NSAID use was not significantly associated with eGFR decline in patients with a normal or mildly impaired eGFR or in those with stage 3-5 CKD at baseline (table 6). Moreover, there was no trend suggesting an increase in the risk of eGFR decline between the normal and high dose user groups.

High dose aspirin users with a normal or mildly impaired eGFR at baseline had a significantly lower risk of eGFR decline than non-users; OR $=0.52$ (95\% CI 0.35 to 0.77 ), but there was no significant change in eGFR decline in those with normal dose use $(\mathrm{OR})=0.96(95 \%$ CI 0.81 to 1.14). Normal or high-dose aspirin use was not significantly associated with eGFR decline in patients with stage 3-5 CKD at baseline with similar risk estimates between both aspirin dose groups.

Normal or high dose paracetamol use was not significantly associated with eGFR decline in patients with a normal or mildly impaired eGFR or stages 3-5 CKD at baseline. The risk estimates between normal and high dose paracetamol users remained approximately congruent in both CKD subgroups.

\section{DISCUSSION}

\section{Summary}

Normal or high dose NSAID or paracetamol prescribing and normal dose aspirin prescribing over 2 years did not significantly affect the risk of eGFR decline among general practice patients with well-preserved or impaired renal function. However, high dose aspirin prescribing was associated with a reduced risk of eGFR decline in patients with well-preserved renal function but not in those with impaired renal function.

\section{Strengths and limitations}

In this study, drug prescribing was standardised using the WHO defined daily dose method and patients were

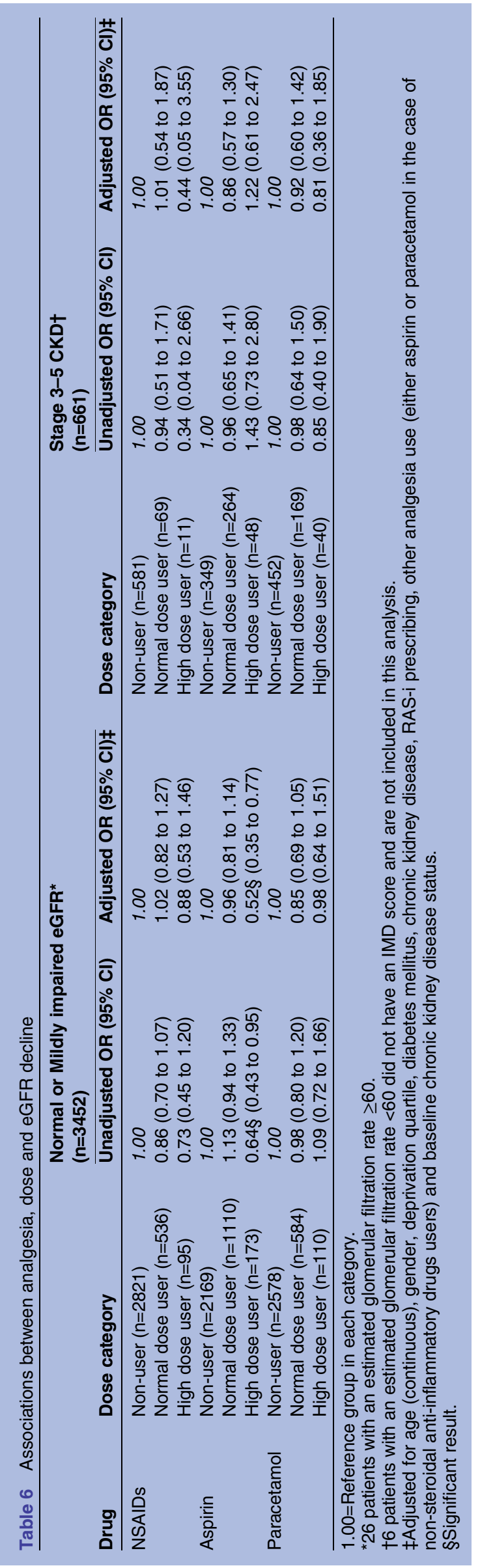


categorised into normal and high-dose groups using and extending the approach set out by Gooch et al, ${ }^{16}$ including both aspirin and paracetamol. The eGFR decline rate was calculated using spaced eGFR intervals and changes were correlated directly against analgesia dose prescribing. Included patients had eGFRs representative of a wide spectrum of CKD and non-CKD. The effect of analgesia use on eGFR decline was stratified into preserved and impaired renal function groups. Finally, the outcome of eGFR decline was based on the NICE and KDIGO guidelines; hence, the results are applicable to current clinical practice. ${ }^{2} 17$

Study limitations are that the number of patients prescribed high doses of analgesics among patients with stage 3-5 CKD at baseline was limited, which may lead to a lack of significance in associations. Over-the-counter (OTC) analgesia use data was not available, but it is likely that long-term users of analgesia are prescribed by GPs and there is evidence that older patients are more likely to have prescribed analgesics. ${ }^{22}$ Key cardiometabolic conditions were included, but other conditions (eg, gout) which may be associated with eGFR decline may influence the associations. However, the effect is likely to be minimal as no significant association between NSAID use and eGFR decline was found and these other conditions are much lower in prevalence. The prescribed analgesics were assumed to have been taken by the patient; hence they did not account for non-compliance. However, the effect of non-compliance is likely to be limited as patients would wish to alleviate painful symptoms and limit the risk of CVD. As exposure prior to 1 January 2009 was not measured, it may be a case where some patients may have had longer exposure to analgesics. In this study, we did not differentiate between new and pre-existing CKD. There is a small risk of misclassification bias as a second eGFR measurement 3 months from the first was not used to confirm baseline stage $3-5$ CKD. $^{1}$ An eGFR decline of $>5 \mathrm{~mL} / \mathrm{min} /$ $1.73 \mathrm{~m}^{2}$ per year has a proportionally greater clinical effect on patients with stage 4 or 5 CKD than in those with preserved renal function, but $93 \%$ of patients within the CKD defined group had stage 3 renal impairment. The study design allowed the investigation of short-term eGFR decline within a 2-year time period but further study would be required to investigate the longterm effects of analgesia prescribing. Finally, use of only the first and last eGFR measurements to calculate eGFR decline may be susceptible to some inaccuracy, given that biological and measurement variation is possible when measuring serum creatinine. This risk is minimised as the outcome chosen in this study would be clinically relevant and not merely due to baseline variation. ${ }^{12}$

\section{Comparison with existing literature}

NSAIDs and eGFR decline

Normal or high dose NSAID prescribing in our study was not significantly associated with eGFR decline within a 2-year time-period. The findings are consistent with large published studies by Yarger et $a l^{20}$ Gooch et $a l^{16}$ Hemmelgarn et al, ${ }^{23}$ Curhan et al, ${ }^{8}$ Kurth et $a l^{19}$ and Rexrode et $a l^{21}$ who found no association between normal dose NSAID use (prescribed or OTC) and renal decline. Equally, a recent systematic review by Nderitu et $a l^{4}$ found no significant association between normal dose NSAID use and eGFR decline. Moreover, studies by Curhan et $a l^{8}{ }^{8}$ Kurth et $a l^{19}{ }^{19}$ Rexrode et $a l^{21}$ found no association between high dose NSAID use and eGFR decline.

In contrast to our study, Gooch et $a l^{16}$ found a significant association between high dose NSAID use and rapid eGFR decline. The contradiction to our findings may be due to the younger participants (mean age 66 vs 76) ${ }^{16}$ and limited number of patients with stage 3-5 CKD prescribed high dose NSAIDs in this study. It is also not possible to comment on whether there were differences in the patterns of NSAID use (continuous vs non-continuous) between study populations, which may explain some of the differences in eGFR decline. Finally, the 2-year period may not have been long enough for the chronic effects of high dose NSAID use on eGFR decline to become apparent.

\section{Aspirin and eGFR decline}

Normal dose aspirin use in our study did not significantly influence eGFR decline. Similarly, Curhan et al, ${ }^{8}$ and Rexrode et $a l^{21}$ found no significant association between aspirin use and renal function decline. High dose aspirin use was associated with a decreased risk of eGFR decline in patients with well-preserved renal function, but not in those with stage 3-5 CKD. Equally, Evans et $a l^{10}$ found that regular low-dose aspirin users with advanced CKD had a slower eGFR decline rate than non-users. Furthermore, Kurth et al, ${ }^{19}$ found that aspirin users without cardiovascular risk factors were significantly less likely to have eGFR decline when compared to non-users of aspirin.

However, in Evans et al's ${ }^{10}$ study, aspirin users had advanced CKD, which differs from the high-dose aspirin users in this study who had well-preserved renal function. Differences in the study findings may be due to the fact Evans et al's ${ }^{10}$ study had a longer follow-up period (5-7 years) which may have allowed the effects of longer term of aspirin use on eGFR decline to become evident in patients with poor renal function. Regular aspirin users in Evans et al's ${ }^{10}$ study were categorised according to frequency of use over a 2-week period before inclusion, unlike in this study where the DDD was used. Differences in patterns and definitions of aspirin use between the two studies may explain differences in outcomes.

It is evident that in patients with CVD, aspirin is renoprotective due to the reduction in GVD. ${ }^{17}$ However, models in this study were adjusted for the presence of CVD, which included codes for hypertensive disease, ischaemic heart disease, heart failure, dysrhythmia, 
cerebrovascular disease and peripheral arterial disease. High-dose aspirin users had an average intake of approximately $110 \mathrm{mg}$ per day which in common clinical practice falls within the thromboprophylactic lowmedium dose use category. Therefore, the reduction in the risk of eGFR decline may be independent of the cardioprotective action. Similarly, this possible renoprotective action was evident in Kurth et al's ${ }^{19}$ study in patients free from CVD risk factors. Models in Evans et al's study were not adjusted for the presence of CVD;hence, the renoprotection may have been secondary to the cardiovascular protective actions of aspirin. Overall, the evidence is unclear as to whether aspirin is renoprotective, independent of its cardioprotective role, and further study is required to explore this potentially clinically useful effect.

\section{Paracetamol and eGFR decline}

Normal or high dose paracetamol prescribing in our study was not significantly associated with eGFR decline at any CKD stage. Similarly, most studies have found no association between paracetamol use and renal dysfunction or progression at any dose. 8 10 1921 However, Curhan $e t a l^{8}$ found that patients with high dose paracetamol use $(>500 \mathrm{~g})$ had an increased risk of eGFR decline. However, this finding was not mirrored by a similar study by Kurth et $a l,{ }^{19}$ even at very high doses of paracetamol (>2000 g). Both studies use different definitions of high-dose paracetamol use (in grams), as opposed to the DDD definitions used in this study, which may explain the differences in observed outcomes. The majority of the published evidence indicates no significant association between paracetamol use and eGFR decline.

\section{CONCLUSIONS}

In conclusion, NSAID, aspirin and paracetamol prescribing were not significantly associated with an increased risk of eGFR decline over a 2-year time-period in patients with well-preserved renal function or stage 3-5 CKD. High dose aspirin prescribing was associated with a decreased risk of eGFR decline in patients with wellpreserved renal function.

NSAIDs use can lead to acute renal dysfunction and gastrointestinal bleeding; hence, they should be prescribed with caution at the lowest effective dose with annual renal function monitoring. Paracetamol prescribing appears to be safe in patients with CKD. Aspirin prescribing does not increase the risk of eGFR decline but future studies should explore the possible significance of the association between aspirin use and a decreased risk of eGFR decline. Future studies should also explore the long-term effects of OTC and prescribed analgesia use on eGFR decline.

Acknowledgements The authors thank the Keele GP Research Partnership and Informatics team at the Arthritis Research UK Primary Care Centre, all the patients and general practitioners for all their contributions to this study.
Contributors PN, SJD and UTK conceived the idea for and design of the study. PN, LD and VYS carried out the analysis of the data. PN drafted the manuscript. All authors were involved in the interpretation of data, critical revision of the manuscript and approved the final version for submission.

Funding This work was supported by the Wolfson Foundation (intercalation award to PN) and the North Staffordshire Medical Institute (academic funding to PN); CiPCA is funded by the North Staffordshire Primary Care Research Consortium and Keele University Institute for Primary Care and Health Sciences

\section{Competing interests None.}

Provenance and peer review Not commissioned; externally peer reviewed.

Data sharing statement No additional data are available.

Open Access This is an Open Access article distributed in accordance with the Creative Commons Attribution Non Commercial (CC BY-NC 4.0) license, which permits others to distribute, remix, adapt, build upon this work noncommercially, and license their derivative works on different terms, provided the original work is properly cited and the use is non-commercial. See: http:// creativecommons.org/licenses/by-nc/4.0/

\section{REFERENCES}

1. National Kidney Foundation. K/DOQI clinical practice guidelines for chronic kidney disease: evaluation, classification and stratification. Am J of Kidney Dis 2002;39(Suppl 1):S1-266.

2. The National Collaborating Centre for Chronic Conditions. Chronic kidney disease. National clinical guideline for early identification and management in adults in primary and secondary care. London: Royal College of Physicians, 2008.

3. Huerta C, Castellsague J, Varas-Lorenzo C, et al. Nonsteroidal anti-inflammatory drugs and risk of ARF in the general population. Am J Kidney Dis 2005;45:531-9.

4. Nderitu P, Doos L, Jones PW, et al. Non-steroidal anti-inflammatory drugs and chronic kidney disease progression: a systematic review. Fam Pract 2013;30:247-55.

5. Bhopal S, Chan J, Ellis O, et al. Non-steroidal anti-inflammatory drugs prescribing in chronic kidney disease: an observational study. Prim Health Care Res Dev 2010;11:280-4.

6. BMA, RPSGB. British National Formulary No.62. London: BM Publishing Group, 2011

7. Coxib and traditional NSAID Trialists' (CNT) Collaboration. Vascular and upper gastrointestinal effects of non-steroidal anti-inflammatory drugs: meta-analyses of individual participant data from randomised trials. Lancet 2013;382:769-79.

8. Curhan GC, Knight EL, Rosner B, et al. Lifetime nonnarcotic analgesic use and decline in renal function in women. Arch Intern Med 2004:164:1519-24.

9. Fored CM, Ejerblad E, Lindblad P, et al. Acetaminophen, aspirin, and chronic renal failure. N Engl J Med 2001;345:1801-8.

10. Evans M, Fored CM, Bellocco R, et al. Acetaminophen, aspirin and progression of advanced chronic kidney disease. Nephrol Dial Transplant 2009;24:1908-18.

11. Jordan K, Clarke AM, Symmons DPM, et al. Measuring disease prevalence: a comparison of musculoskeletal disease using four general practice consultation databases. Br J Gen Pract 2007;57:7-14.

12. Jordan K, Jöud A, Bergknut C, et al. Extended report: International comparisons of the consultation prevalence of musculoskeletal conditions using population-based healthcare data from England and Sweden. Ann Rheum Dis 2014;73:212-18.

13. Porcheret $M$, Hughes $R$, Evans $D$, et al. Data quality of general practice electronic health records: the impact of a program of assessments, feedback, and training. $J$ Am Med Inform Assoc 2004:11:78-86.

14. de Lusignan $\mathrm{S}$, Chan $\mathrm{T}$, Stevens $\mathrm{P}$, et al. Identifying patients with chronic kidney disease from general practice computer records. Fam Pract 2005;22:234-41.

15. WHO collaborating centre for drug statistics methodology. Anatomical Therapeutic Chemical (ATC) index with Defined Daily Doses (DDDs) [Online]. http://www.whocc.no/atcddd/ (accessed Feb 2012).

16. Gooch K, Culleton BF, Manns BJ, et al. NSAID use and progression of chronic kidney disease. Am J Med 2007;120:280.e1-e7.

17. KDIGO. Clinical practice guideline for the evaluation and management of chronic kidney disease. Kidney Int Supp/ 2012;3: $5-119$. 
18. McLennan $\mathrm{D}$, Barnes $\mathrm{H}$, Noble $\mathrm{M}$, et al. The indices of deprivation 2010 (ID 2010). Dept Commun Local Govt 2011;3:8-50.

19. Kurth T, Glynn RJ, Walker AM, et al. Analgesic use and change in kidney function in apparently healthy men. Am J Kidney Dis 2003;42:234-44.

20. Yarger S, Nwokeji E, Trice S, et al. Puk4 Cumulative exposure to nonsteroidal anti-inflammatory drugs (NSAIDS) and the progression of chronic kidney disease (CKD) [abstract]. Value in Health 2011;14:A74-5.
21. Rexrode KM, Buring JE, Glynn RJ, et al. Analgesic use and renal function in men. JAMA 2001;286:315-21.

22. Turunen J, Mantyselka $\mathrm{P}$, Kumpusalo $\mathrm{E}$, et al. Frequent analgesic use at population level: prevalence and patterns of use. Pain 2005; 115:374-81.

23. Hemmelgarn BR, Culleton BF, Ghali WA. Derivation and validation of a clinical index for prediction of rapid progression of kidney dysfunction. QJM 2007;100:87-92. 